\title{
Versatile wide angle diffraction setup for simultaneous wide and small angle $x$-ray scattering measurements with synchrotron radiation
}

\author{
D. R. Rueda, M. C. García-Gutiérrez, A. Nogales, M. J. Capitán, and T. A. Ezquerra ${ }^{a}$ \\ Instituto de Estructura de la Materia, CSIC, Serrano 119, 28006 Madrid, Spain
}

A. Labrador, E. Fraga, D. Beltrán, J. Juanhuix, J. F. Herranz, and J. Bordas

LLS, BM16-ESRF, 6 rue Jules Horowitz, BP220, 38043 Grenoble, France

(Received 3 January 2006; accepted 5 February 2006; published online 22 March 2006)

\begin{abstract}
Here we present a novel, simple, and versatile experimental setup aimed to perform wide angle $\mathrm{x}$-ray scattering (WAXS) measurements alone or in simultaneous combination with small angle $\mathrm{x}$-ray scattering measurements. The design of the WAXS goniometer allows one to obtain high resolution diffraction patterns in a broad angular range. The setup can incorporate a hot stage in order to evaluate temperature resolved experiments. The performance of the equipment has been verified in the BM16 beam line of the European Synchrotron Radiation Facility with different well known samples such as alumina, isotropic film of high density polyethylene (HDPE), and oriented HPDE fiber. () 2006 American Institute of Physics. [DOI: 10.1063/1.2182806]
\end{abstract}

\section{INTRODUCTION}

Soft condensed matter, in general, includes a great variety of complex materials which frequently present a hierarchical structure ranging from tenths to hundreds of nanometers. ${ }^{1} \mathrm{X}$-ray diffraction is a powerful tool to characterize the structure of complex materials at the different length scales. ${ }^{2}$ Simultaneous measurements of wide and small angle X-ray scatterings, WAXS and SAXS, respectively, have been shown to be of great importance while dealing with the structural analysis of soft condensed matter ${ }^{2}$ because structural characterization of different building blocks of a complex material can be obtained. ${ }^{2,3}$ Of especial interest is when simultaneous SAXS and WAXS are combined with temperature ${ }^{4}$ variations and eventually with a third experimental technique. ${ }^{5,6}$ Such a combination of techniques provides information to investigate the sample phase behavior. The traditional WAXS and SAXS setup involves the combination of either a pair of one-dimensional detectors ${ }^{6}$ or a one-dimensional detector for WAXS and a two-dimensional detector for SAXS. ${ }^{45}$ The use of a couple of two-dimensional detectors for SAXS and WAXS, although available, ${ }^{7}$ has been less demanded. In any case the WAXS detector is aligned at a fixed $2 \theta$ angle in relation to the sample position. In this way a variation of the angular detection range is obtained by changing the sample-detector distance. This setup is very useful for most of the experimental situations, however, the detection of higher $2 \theta$ angles is excluded. For this reason and, in particular, when high resolution is required, the possibility of both the $2 \theta$ region selection and a long sample-detector distance seems to be quite appealing.

In the present article, we describe a new and simple setup for simultaneous WAXS and SAXS measurements with synchrotron radiation which increases the versatility in

${ }^{a)}$ Electronic mail: imte155@iem.cfmac.csic.es the WAXS detection range by allowing both access to higher $2 \theta$ angular region and variable detection resolution.

\section{EXPERIMENTAL SETUP}

\section{A. Source characteristics}

The setup described below was placed at the BM16 beam line of the European Synchrotron Radiation Facility (ESRF), Grenoble, France. BM16 uses radiation from one of the ESRF bending magnets with a critical energy of $19.6 \mathrm{keV}$. The optics is based on a flat vertically collimating mirror, a double crystal monochromator $\mathrm{Si}(111)$, and a toroidal mirror. The optics is designed to provide an optimized flux and focal spot in the $6-17 \mathrm{keV}$ energy range. In the present case an energy of $12.4 \mathrm{keV}$ corresponding to a wavelength of $\lambda=0.1 \mathrm{~nm}$ was used. A detailed description of the BM16 layout has been presented elsewhere. ${ }^{8}$

\section{B. Description of the experimental setup}

Figure 1 shows a scheme of the experimental setup aimed for the simultaneous WAXS and SAXS measurements. The whole setup consists of the following elements:

- One circle goniometer which includes a rotation unit RV240PE from Newport (1) and a supporting arm (2) manufactured by RAMEM S.A. ${ }^{9}$ This arm holds at one end the charge coupled device (CCD) detector and at the other end a counterweight element (3) to balance for the detector weight. The sample environment is large enough to fit a Linkam hot stage (4) for thermal experiments.

- The WAXS detector is a CCD camera PI SCX90-1300 from Roper Scientific. The active surface is 65 $\times 65 \mathrm{~mm}^{2}$ and has $1242 \times 1152$ pixels.

- A vacuum chamber consisting of modular stainless steel elements which can be selected to provide the required 


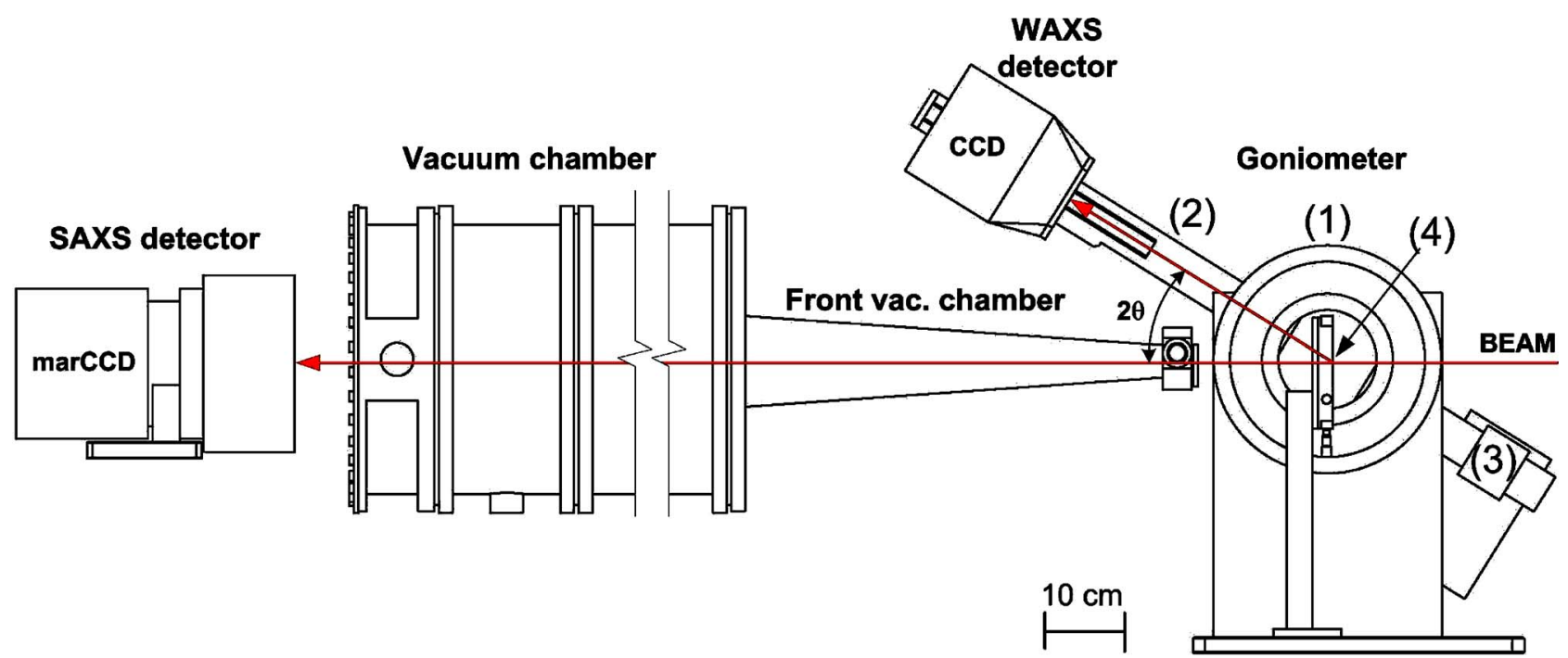

FIG. 1. Scheme of the experimental setup for simultaneous SAXS-WAXS experiments at the BM16 beam line. The main parts of the goniometer are rotation unit (1), arm (2), counterweight (3), and hot stage (4). The vacuum chamber is made of cylindrical parts of different lengths and a square based truncated pyramid at the front.

sample to SAXS-detector distance. In the present case the distance was fixed to $2.5 \mathrm{~m}$.

- A front vacuum chamber unit consisting of a hollow pyramid of square basis which is truncated at the end. This unit is sealed in the front part by a thin Kapton foil pressed against the outer surface by a metallic frame with an o ring and screws. The back part is fused to a standard stainless steel flange (MF, Blank, NW 320, 304) to connect it to the vacuum chamber. The angle between the upper surface of the front unit and the beam direction is $5^{\circ}$. This aperture is wide enough to allow the passage of diffracted intensity in the SAXS region without significant shadowing.

- A SAXS detector which for this case was a marCCD from X-Ray Research, GmbH, Norderstedt, Germany. The detector has an active surface of $165 \mathrm{~mm}$ diameter and $2048 \times 2048(2 \times 2$ binned $)$ pixels.

The goniometer is mounted with its rotation axis perpendicular to the beam direction. The sample position is located in the intersection between the goniometer axis and the beam. The goniometer arm rotation is such that the CCD detector rotates in the vertical plane containing the beam direction. The detection plane of the CCD detector is always maintained perpendicularly to the radius defined by the arm. The CCD detector can be displaced continuously to vary the distance $(D)$ to the sample between 20 and $40 \mathrm{~cm}$. This allows for different angular resolutions in WAXS as it is shown in the following examples.

\section{WAXS calibration}

Standard of alumina, $\mathrm{Al}_{2} \mathrm{O}_{3}$, was used for the WAXS calibration. The analysis of the WAXS images was accomplished by the POLAR 2.6.9 software package (Stony Brook Technology and Applied Research, Inc.). The WAXS pattern for alumina taken at the minimum allowed $2 \theta$ angle and for a sample-detector distance of $20 \mathrm{~cm}$ is shown in Fig. 2. One horizontal line profile is valid to measure the peak position of the observed reflections, however, in order to improve the signal-noise ratio of the intensity profiles, an integration of the central part of the reflections was accomplished. The angular sector for the integration was from $-5^{\circ}$ to $5^{\circ}$ around the axis $(y=700)$ containing the beam center, which in this case was defined at the pixel coordinates $(-1000,+700)$, i.e., out of the image. The inset in Fig. 3 presents $s$ vectors as a function of the pixel position for the four reflections showing an acceptable linearity between both magnitudes. Figure 3 shows the corresponding integrated intensity profiles versus their reciprocal lattice spacing values, $s=2 / \lambda(\sin \theta)$. The ratio between the different peak maxima is similar to that observed by standard diffractometry, thus supporting the validity of our integration procedure.

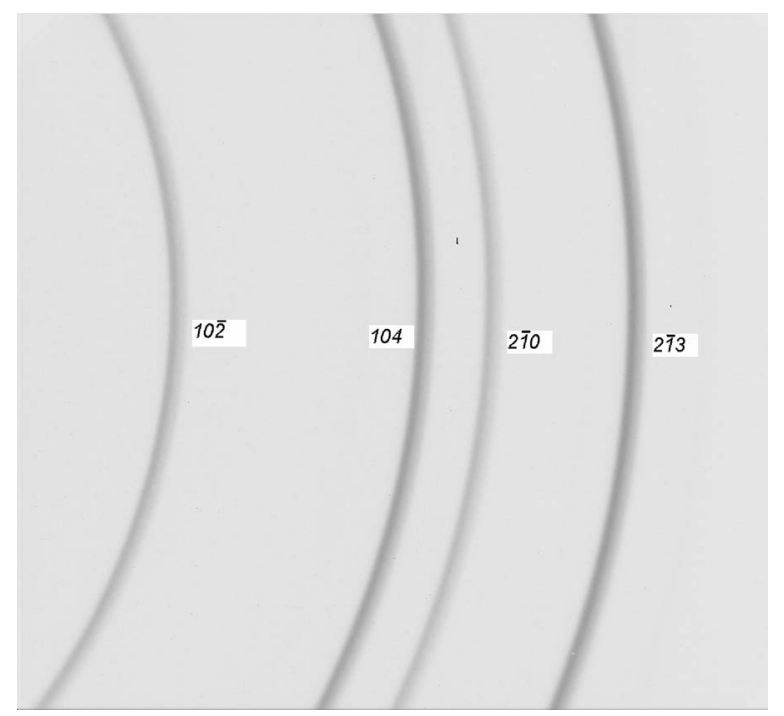

FIG. 2. WAXS pattern of alumina obtained in the low resolution arrangement $(D=20 \mathrm{~cm})$, at $2 \theta_{\min }=22.0^{\circ}$ and with an accumulation time of $30 \mathrm{~s}$. 


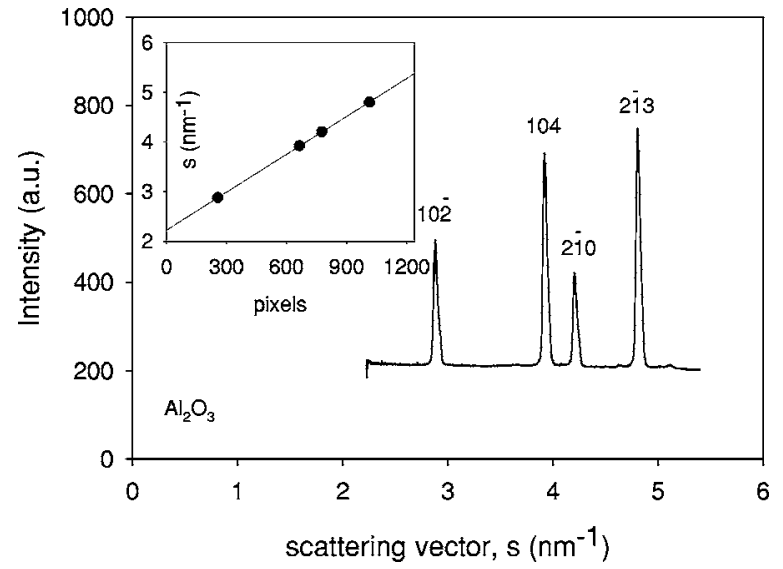

FIG. 3. WAXS intensity profile for alumina derived from the $\mathrm{x}$-ray pattern of Fig. 2. The inset shows the calibration curve $\left[s\left(\mathrm{~nm}^{-1}\right)=2.2225+2.545\right.$ $\times 10^{-3} n$ (pixel)] obtained for alumina using the low resolution setup.

\section{Versatile WAXS recording}

\section{Isotropic samples}

The goniometer arm is provided with a millimeter scale to indicate the CCD-detector position in relation to the goniometer axis and therefore to the sample. In order to test the angular resolution of the diffraction patterns at different detector positions the two extreme sample-detector distances, schematized in Fig. 4, were selected. The shortest position $[D=20 \mathrm{~cm}$, Fig. 4(a)] is referred to as the low resolution

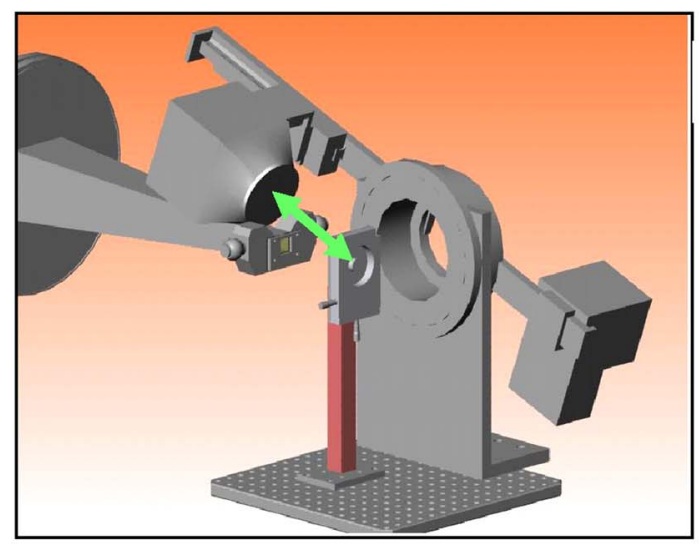

(a)

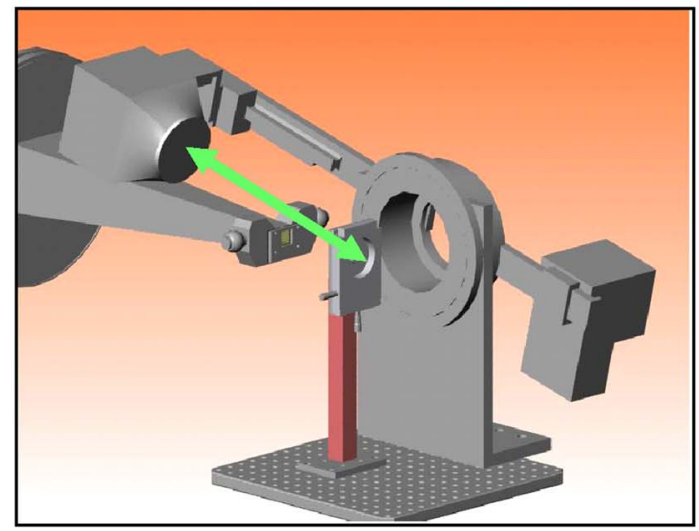

(b)

FIG. 4. Scheme showing the two extreme sample-detector distances. The shortest position $[D=20 \mathrm{~cm}$, (a) $]$ is referred to as the low resolution position and the largest one $[D=40 \mathrm{~cm},(\mathrm{~b})]$ is referred to as the high resolution position.

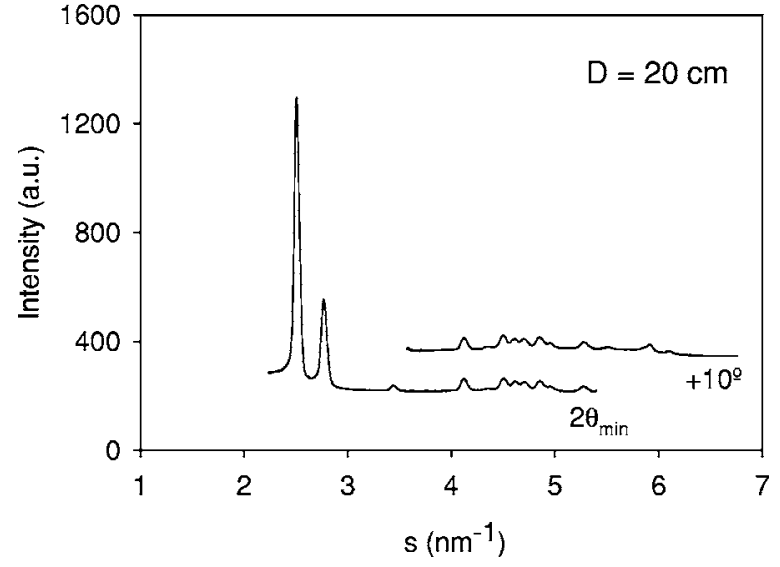

FIG. 5. WAXS intensity profiles as a function of the scattering vector $s$ for HDPE obtained in the low resolution arrangement $(D=20 \mathrm{~cm})$ at $2 \theta_{\text {min }}$ $=22.0^{\circ}$ and $10^{\circ}$ above. Diffractograms are vertically shifted for clarity.

position and the largest one $[D=40 \mathrm{~cm}$, Fig. 4(b) $]$ is referred to as the high resolution position. A high density polyethylene (HDPE) (Lupolene) sample was used as standard. WAXS images were obtained in angular steps of $5^{\circ}$ starting from the minimum angle accessible, $2 \theta_{\min }$, obtained when the WAXS detector is in direct contact with the upper flat surface of the front part of the vacuum chamber. The $2 \theta_{\min }$ values for the low and high resolution positions are $22.0^{\circ}$ and $13.7^{\circ}$, respectively. The integrated radial profiles of the WAXS patterns versus the $s$ vector are shown in Figs. 5 and 6 for the low and high resolution cases, respectively. Figure 5 only shows two profiles corresponding to $2 \theta_{\min }$ and $2 \theta_{\min }$ $+10^{\circ}$ because of the large overlapping of consecutive profiles. The intensity profiles obtained present the characteristics of a typical HDPE diffractogram. ${ }^{10}$ The different angular resolutions achieved in the two extreme detector positions are remarkable. Thus, for low resolution $(D=20 \mathrm{~cm})$ one CCD image covers an $s$ interval of $3.2 \mathrm{~nm}^{-1}$ which is about twice of that recorded for high resolution $\left(\Delta s=1.51 \mathrm{~nm}^{-1}\right)$. This is clearly seen from the different portions of the PE diffractogram shown in the intensity profiles of Figs. 5 and 6. The difference in the smallest $s$ values achieved for the low and high resolution cases is also worth mentioning. The

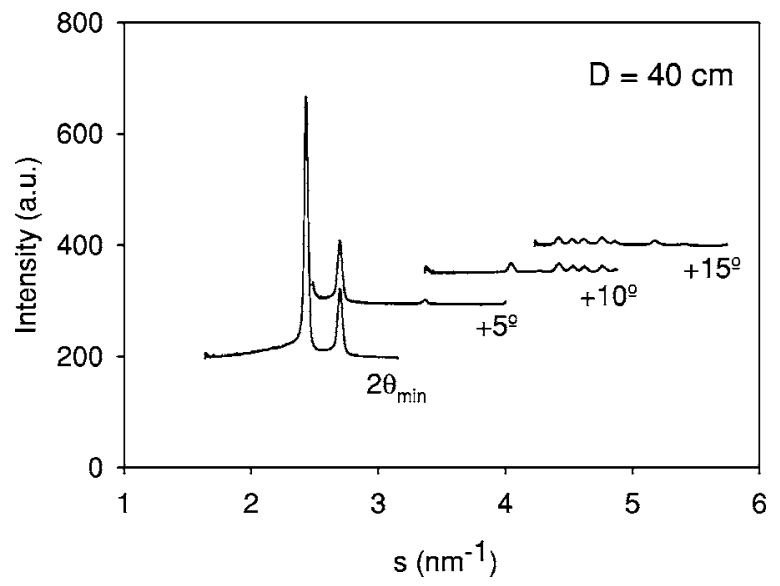

FIG. 6. WAXS intensity profiles of HDPE obtained in the high resolution disposition $(D=40 \mathrm{~cm})$ in angular steps of $5^{\circ}$ starting from $2 \theta_{\min }=13.7^{\circ}$. Diffractograms are vertically shifted for clarity. 


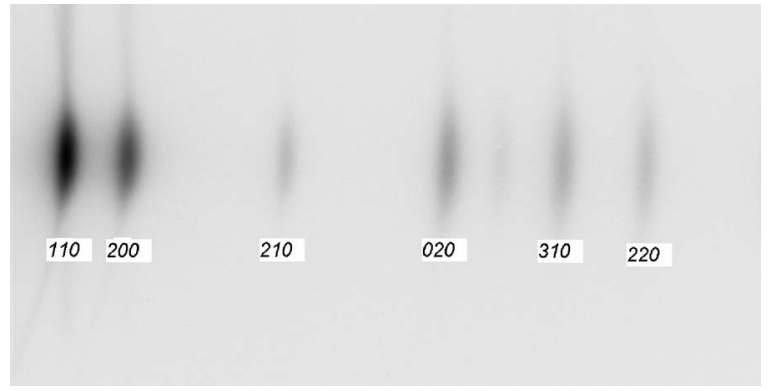

FIG. 7. WAXS pattern of a HDPE fiber horizontally mounted perpendicular to the beam and parallel to the rotation axis $(D=20 \mathrm{~cm}$, accumulation time of $10 \mathrm{~s}$ ) showing the $h k 0$ reflections.

minimum $s$ value to be recorded in this WAXS setup and with a wavelength of $0.1 \mathrm{~nm}$ is $1.7 \mathrm{~nm}^{-1}$ which corresponds to lengths of $\approx 0.6 \mathrm{~nm}$. The loss of peak intensity observed for the high resolution case with respect to that observed in the low resolution one is explained by the air absorption of scattered intensity. It is worth noticing that the goniometer arm can rotate up to higher angles, even above $90^{\circ}$. This could be of interest to study inorganic materials or molecular compounds showing high angle reflections of interest.

\section{Oriented samples}

In the case of oriented samples, for example, polymer fibers, the two-dimensional detection in both WAXS and SAXS is very helpful to elucidate their structure and morphology. Usually polymer fibers exhibit uniaxial orientation around the macroscopic fiber axis. ${ }^{11}$ Therefore, with the WAXS configuration of Fig. 1, two different fiber orientations, horizontal and vertical, can be easily proven. For example, if a polyethylene fiber is horizontally mounted (i.e., perpendicular to the beam and parallel to the rotation axis) then the $h k 0$ reflections can be recorded by the WAXS detector (Fig. 7). The corresponding SAXS pattern is not presented because the current size of the beam stop hides SAXS information in this case. However, if the fiber is vertically mounted (i.e., perpendicular to the beam and to the rotation axis) then the oriented SAXS maximum characteristics of a polyethylene fiber ${ }^{11}$ are clearly resolved (Fig. 8). Additionally the 001 reflections can be easily recorded provided the goniometer arm is moved to the appropriate $2 \theta$ angular position.

\section{E. Possibility of temperature resolved simultaneous WAXS-SAXS detection}

The setup presented in Fig. 1 can easily house a hot stage for temperature resolved experiments. A Linkam THMS600 from Linkam Scientific Instruments Ltd. was installed and integrated in the data acquisition control for that purpose. For these measurements, the two glass windows of the hot stage were replaced by $0.025 \mathrm{~mm}$ thick Kapton foils. In the following example again an isotropic HDPE (Lupolene) sample of about $0.3 \mathrm{~mm}$ thick was wrapped with aluminum foil in the sample holder of the hot stage in order to prevent sample losses by flow above the melting temperature. Figure 9 shows the two-dimensional WAXS (top) and SAXS (bottom) patterns, respectively, recorded at room tem-

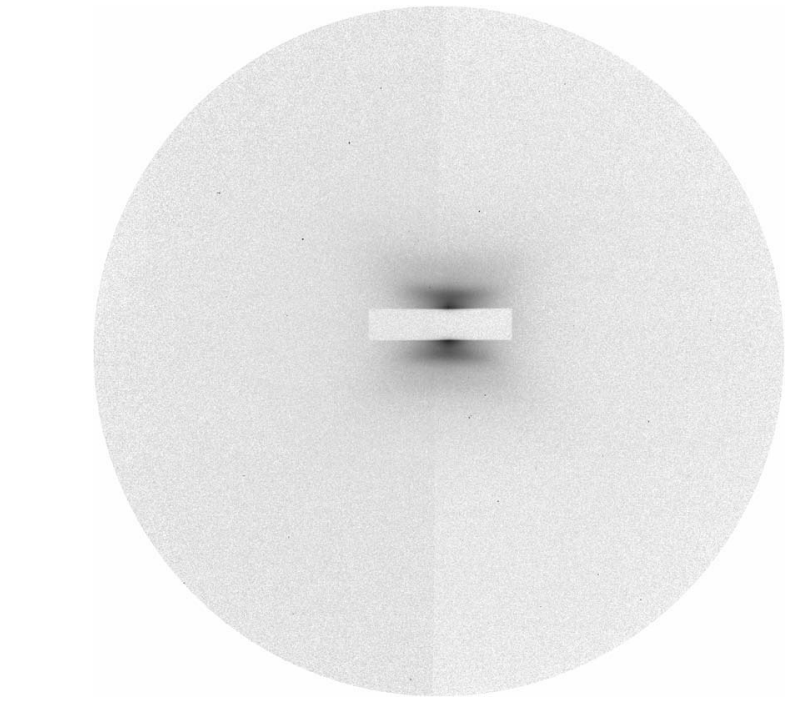

FIG. 8. SAXS pattern of a HDPE fiber vertically mounted perpendicular to the beam and the rotation axis $(D=20 \mathrm{~cm}$, accumulation time of $10 \mathrm{~s})$.

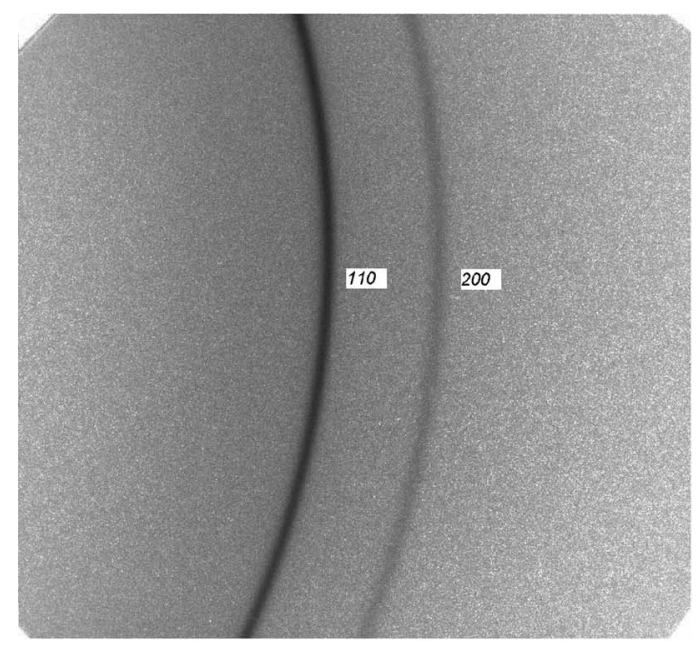

(a)

(b)

FIG. 9. (a) WAXS and (b) SAXS patterns of HDPE at room temperature after background subtraction. The sharp outer reflection in the SAXS pattern arises due to the paraffin wax fraction of polyethylene $(D=40 \mathrm{~cm}$, accumulation time of $30 \mathrm{~s}$ ). 

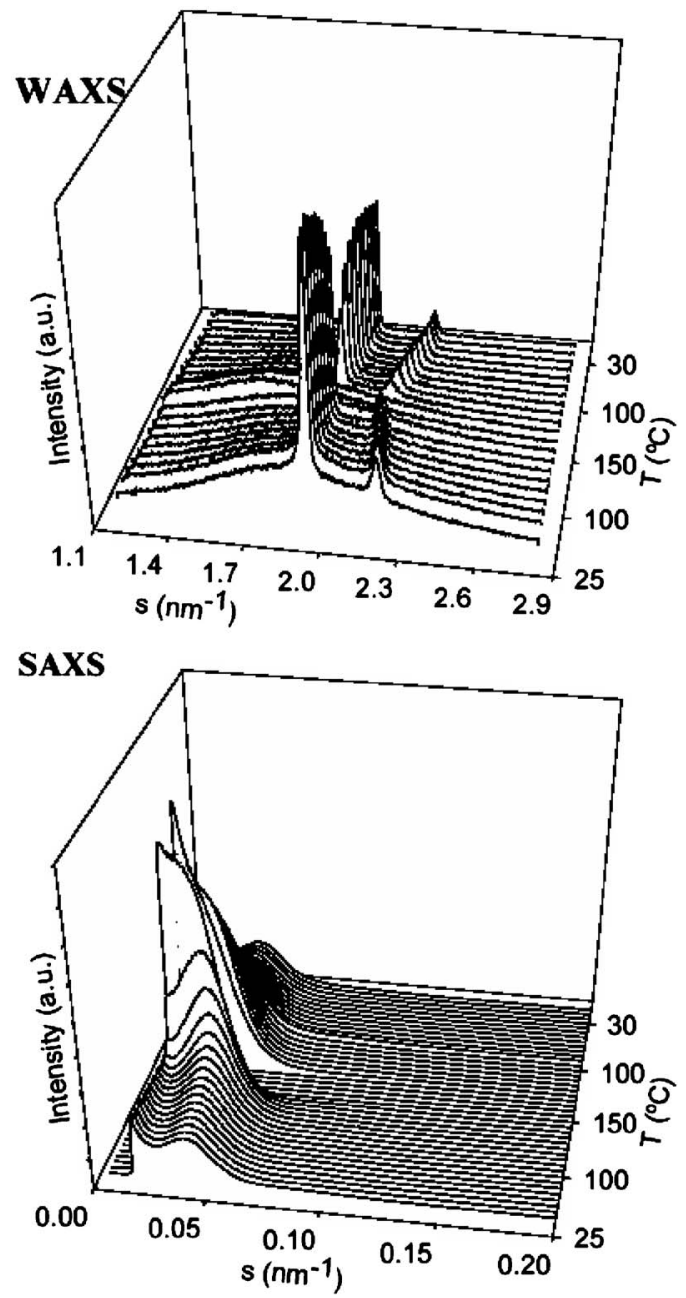

FIG. 10. Three-dimensional plots illustrating the melting and recrystallization process for HDPE. The process was followed by simultaneous WAXS (top) and SAXS (bottom) measurements. The diffracted intensity has been represented as a function of both the $s$ vector and the temperature.

perature after subtraction of the empty holder images (background). Silver behenate was the standard used for calibration and determination of the actual center in the SAXS patterns. A radial integration between $260^{\circ}$ and $280^{\circ}$ (to avoid interferences from the beam stop) was done to define the SAXS intensity profiles. WAXS patterns were analyzed as described further above. Figure 10 shows the integrated intensity profiles corresponding to a simultaneous WAXS (top) and SAXS (bottom) measurements during a heatingcooling process of the polyethylene sample. The temperature profile, provided by a Linkam TP94 temperature controller, consists on first a heating ramp, at $2{ }^{\circ} \mathrm{C} / \mathrm{min}$, from room temperature to $150{ }^{\circ} \mathrm{C}$. After $1 \mathrm{~min}$ at $150{ }^{\circ} \mathrm{C}$ the temperature was further decreased, at $-2{ }^{\circ} \mathrm{C} / \mathrm{min}$, down to $30^{\circ} \mathrm{C}$. The accumulation time for both SAXS and WAXS patterns was $30 \mathrm{~s}$. In this example the high resolution for WAXS was selected. In this way, the evolution with temperature of the two strongest reflections (110 and 200) of PE as well as the amorphous halo can be optimally analyzed. Figure 10 shows how the melting and crystallization process of polyethylene has been clearly resolved in this experiment.

\section{ACKNOWLEDGMENTS}

The authors are indebted to the Spanish Ministry of Science and Education (Grant No. FPA2001-2139) and to the EU, Marie-Curie reintegration grant (MERG-CT-2004511908) for the financial support of this project. The technical assistance of I. Sanz and J. Granados (CSIC) and of E. Ramiro and M. Sánchez from Ramem S.A. are greatly acknowledged.

${ }^{1}$ R. Jones and R. A. L. Jones, Soft Condensed Matter (Oxford University Press, Oxford, 2002).

${ }^{2}$ Neutrons, X-rays and Light: Scattering Methods Applied to Soft Condensed Matter, edited by P. Lindner and Th. Zemb (North-Holland, Amsterdam, 2002).

${ }^{3}$ Scattering from Polymers: Characterization by X-ray, Neutrons, and Light, ACS Symposium Series 739, edited by P. Cebbe, B. S. Hsiao, and D. Lohse (American Chemical Society, Washington, DC, 2000).

${ }^{4}$ W. Bras and A. J. Ryan, J. Appl. Crystallogr. 30, 816 (1997).

${ }^{5}$ C. Wutz, M. Bark, J. Cronauer, R. Döhrmann, and H. G. Zachmann, Rev. Sci. Instrum. 66, 1303 (1995).

${ }^{6}$ I. Sics, A. Nogales, T. A. Ezquerra, Z. Denchev, and F. J. Balta-Calleja, Rev. Sci. Instrum. 71, 1733 (2000).

${ }^{7}$ A. Mahendrasingam, D. J. Blundell, A. K. Wright, V. Urban, T. Narayanan, and W. Fuller, Polymer 45, 5641 (2004).

${ }^{8}$ J. Juanhuix, A. Labrador, D. Beltrán, J. F. Herranz, P. Carpentier, and J. Bordas, Rev. Sci. Instrum. 76, 861031 (2005).

${ }^{9}$ www.ramem.com

${ }^{10}$ F. J. Baltá-Calleja and C. G. Vonk, X-ray Scattering of Synthetic Polymers (Elsevier, Amsterdam, 1989).

${ }^{11}$ D. R. Rueda, F. Ania, and F. J. Baltá-Calleja, Polymer 38, 2027 (1997). 